\title{
Pour une lecture éco-poétique de Boum et Dog
}

Sylvie Brodziak

\section{OpenEdition \\ Journals}

Édition électronique

URL : https://journals.openedition.org/coma/7378

DOI : $10.4000 /$ coma. 7378

ISSN : 2275-1742

\section{Éditeur}

Institut des textes \& manuscrits modernes (ITEM)

\section{Référence électronique}

Sylvie Brodziak, « Pour une lecture éco-poétique de Boum et Dog», Continents manuscrits [En ligne], 17। 2021, mis en ligne le 15 octobre 2021, consulté le 07 janvier 2022. URL : http:// journals.openedition.org/coma/7378; DOI : https://doi.org/10.4000/coma.7378

Ce document a été généré automatiquement le 7 janvier 2022.

\section{(c) (i) () $\Theta$}

Continents manuscrits - Génétique des textes littéraires - Afrique, Caraîbe, dispora est mis à disposition selon les termes de la licence Creative Commons Attribution - Pas d'Utilisation Commerciale - Pas de Modification 4.0 International. 


\title{
Pour une lecture éco-poétique de Boum et Dog
}

\author{
Sylvie Brodziak
}

\section{Introduction}

1 En étudiant la nouvelle Boum et Dog publié en 1932 dans le numéro 134 de la revue Les Cuvres libres, recueil littéraire mensuel ne publiant que de l'inédit, j'ai été surprise par la façon dont, tout en constatant et décrivant le rapport traditionnel ancestral de domination que l'homme entretient avec l'animal, René Maran tente de le déconstruire en installant l'animal et la nature en souveraineté, et ceci sans passer par une excessive anthropomorphisation de la faune et de la flore. Tout en expliquant la conduite animale par l'instinct et celle de l'homme par la raison, Maran par son style altère la représentation de la hiérarchie entre les espèces. Ainsi, dès 1932, dans cette nouvelle apparait un récit à venir qui suppose une autre relation entre l'homme et l'animal et un autre rapport au vivant.

2 Ma tâche sera donc de démontrer en quoi cette nouvelle soumise à l'éco-critique ${ }^{1}$ appartient à l'éco-poétique à savoir, une littérature qui a non seulement le souci de la nature et de sa préservation mais aussi du "réseau inextricable d'affinités », expression empruntée à Darwin, et reprise à la fois par la philosophe et biologiste américaine Donna Haraway en 2003 dans son Manifeste des espèces compagnes et la philosophe et psychologue belge Vinciane Despret dont les travaux vont particulièrement m'inspirer ${ }^{2}$. J'examinerai également comment ce récit satisfait à l'enjeu de la géopoétique ou production d'une écriture à partir d'un lieu.

\section{Méthodologie}

3 En quoi les travaux de Vinciane Despret, philosophe des sciences et observatrice des pratiques des éthologues, inspirent-ils la littéraire que je suis? Par le fait qu'elle 
considère que les scientifiques pensent, lient, racontent et fictionnalisent les récits produits sans cesse par le monde vivant pour construire le discours de la science.

4 Autrement dit, Vinciane Despret constate que la question de l'écriture (du récit) est au cœur de tout le vivant. Ainsi les animaux et les plantes peuvent écrire des histoires qui ressemblent aux nôtres. Les topoi communs sont IDENTIQUES: des questions de territoire, de frontière, de coopération, de lutte, de goût, de beauté, d'acte de bravoure, d'amour...

5 Mon intention est donc de tenter avec Boum et Dog, d'aller au-delà des descriptions de la faune et la flore, de l'anthropomorphisation des bêtes et de l'inventaire ethno-littéraire des usages et coutumes d'un village observé par un ex-fonctionnaire colonial en Oubangui-Chari.

\section{Géopoétique}

Le caractère géopoétique de cette nouvelle, qui narre l'amitié d'un chien et d'un buffle détruite par les hommes chasseurs, se manifeste dans le mélange de la représentation émerveillée de la nature et le rejet de la figure paradisiaque de l'Afrique.

René Maran ouvre son roman par la brève description d'un paysage au lever du jour que « le Chari et le Logone cernent de leurs méandres ${ }^{3}$ ». Un tel incipit qui situe l'action et plante le décor n'est en rien original. Toutefois, si pendant les années vingt et trente, la représentation littéraire de l'Afrique demeure exotique, elle n'est plus celle d'une terra incognita et l'Europe se délecte de l'image de l'Afrique sauvage, de la wilderness fabriquée par la colonisation, confortée par le récit des explorateurs et des scientifiques. Ainsi, depuis le $\mathrm{XIX}^{\mathrm{e}}$ siècle, époque où poètes et romanciers s'insurgeaient contre les méfaits de l'industrialisation et du capitalisme sauvage (Vigny par exemple ou encore Michelet), l'Afrique brutalement explorée est devenue pour ceux et celles qui en reviennent un pays enchanteur, une terre de merveilles, tel que l'exprime encore en 1907 le professeur agrégé au lycée de Bordeaux, Gabriel Galland qui, à partir du journal de route d'un des membres des deux missions Émile Gentil parties à la conquête du Tchad, compose un ouvrage édifiant destiné à la cérémonie annuelle de la distribution des prix aux « chers écoliers de France »:

Au seuil des cases, à l'entrée des villages, trophées sinistres des crânes humains! Nous sommes bien en pleine anthropophagie. Les seuls rapports que l'on puisse avoir avec de pareilles gens consistent dans les achats. [...] le marché terminé, bravement le vapeur s'éloignait et l'on allait camper plus loin. Avec des gaillards de cette taille et si friands de chair fraîche, on ne saurait prendre trop de précautions. [...] A nouveau, la forêt, végétation splendide, des arbres gigantesques, de cent essences diverses, se reflètent dans les eaux du fleuve; des lianes à caoutchouc, montent, descendent, s'entrecroisent, s'enlacent en inextricables fouillis. Les espèces géantes, les acajous, les ébènes, les fromagers, etc. pointent vers le ciel leurs cimes altières cherchant l'air, la lumière, le soleil, luttant pour se dégager des étreintes des plantes parasites et ces lianes serpents qui les étouffent : c'est une débauche de verdure ${ }^{4}$.

8 Ces images communes à la geste populaire ont nourri sans aucun doute l'imaginaire de Maran $^{5}$, lui qui arrive dans une colonie ratée, qui a du mal à exister, comme le précise Jean-Pierre Tuquoi dans son ouvrage Oubangui-Chari, le pays qui n'existait pas ${ }^{6}$.

9 En effet, lors de son séjour, Maran est amené à constater l'échec de la mission civilisatrice de la France et s'enfonce au cœur de la tragédie humaine, économique et 
écologique que vit la colonie "cendrillon » de l'Empire. Son expérience professionnelle et personnelle de l'Afrique est surtout source de réflexions et de déceptions. Sa façon binaire d'écrire la nature le révèle.

10 Ainsi, le début de Boum et Dog qui est à rapprocher de l'incipit du Livre de la Brousse publié deux ans plus tard est sobre et fort éloigné du traditionnel lyrisme romantique. Malgré cette retenue, tout en affectionnant l'expression cliché "poudroiement d'étoiles ", Maran ne peut s'empêcher de manifester l'éblouissement qu'il éprouve face aux paysages et s'inscrit dans la tradition orphique de la perception de la nature, née au $\mathrm{XVII}^{\mathrm{e}}$ siècle, approche qui manifeste une connaissance empathique avec la nature, source de poésie et d'extase.

11 Cependant, à la différence des écrivains voire des poètes qui l'ont précédé, il privilégie non pas le regard mais les sons, comme l'exprime la phrase suivante : «Souffle du vent, chant timide des perdrix, bruits mouillés de la venue du jour, ricanement d'un protèle, hennissements des chevaux ». Autant de sonorités qui permettent de sortir du système de sens commun ancré dans la culture gréco-latine fondé sur l'œil et la mise à distance.

Le paysage pour René Maran n'est pas un tableau que l'on contemple : « La nature n'est [plus] un temple où de vivants piliers laissent parfois sortir de confuses paroles [et où] les parfums et les sons se répondent ${ }^{7}$.»

13 Le paysage n'est pas symbole, il n'est pas ekphrasis, description poétique des lieux. Il est expérience et s'éprouve, se ressent par tous les pores et tous les sens. Maran donne véritablement chair aux synesthésies, il ne plante pas un décor : il crée une ambiance, une atmosphère sensible.

14 Les sons, les sens et les odeurs sont suggérés non seulement par le lexique employé toujours recherché voire parfois précieux, mais également par le rythme et la musicalité des phrases. Écoutons : "Il faisait froid. Un frisselis infini et indéfinissable emplissait les étendues incertaines des plantes ou du murmure de ses remous"; l'allitération en $s$ nous donne des frissons.

15 Plus explicite encore sensoriellement, un lever du jourdans la brousse, auquel nous assistons étape par étape, et que nous ressentons à la fois par la vue, le toucher et l'odorat :

-étape $1:$ :La brousse sécrétait des brouillards »; l'allitération en $r$ suggère l'air chargé d'humidité de la nuit.

- étape $2:$ :Laube la niellait de rosée»; au lever du jour, la brousse habillée par les gouttelettes de rosée. C'est la rencontre des voyelles $a$ et $i$, et le son [11] qui enveloppe.

- étape 3 :«Le vent gorgé d'aromates souffletait de touffes de parfums les arbres pavoisés de feuilles », les allitérations en $f$ sont expression du souffle mais aussi exhalaison des odeurs.

La narration est acoustique parce que l'Afrique impose un changement du système de sens. Elle le contraint à faire de la géographie sonore et olfactive, et propose au lecteur une nouvelle approche poétique de la Terre, appréhendée en l'occurrence comme Gaïa, à savoir selon Bruno Latour, une figure multiple, qui interagit avec l'ensemble des vivants - les Terrestres - qui la composent. Gaïa est vivante ; elle est réactive, sensible, " extrêmement chatouilleuse ${ }^{8}$ ».

17 En conséquence, en ajoutant à ce concept latourien celui du nouvel âge écologique qu'est l'anthropocène qui met en exergue l'idée des conséquences néfastes perpétrées par les actions des hommes sur la terre, la description poétique devient une poïétique, une esthétique attentive aux percepts et aux affects qui imprègnent les milieux de vie, 
une praxis poétique qui alarme, émeut, peut mettre en mouvement, voire appelle à l'action.

Dans Boum et Dog, l'action s'incarne dans le refus de transmettre le mythe de l'Afrique édénique sauvée par les Blancs. En effet, lorsque le fonctionnaire Maran arrive en Oubangui-Chari, le mythe des forêts "primaires » est déjà répandu. Construit en AOF, par les botanistes français au début du $\mathrm{Xx}^{\mathrm{e}}$ siècle, il se répand en AEF comme l'explique l'historien Guillaume Blanc dans son ouvrage L'Invention du colonialisme vert ${ }^{9}$. Ce mythe consiste à véhiculer l'idée que ce sont les indigènes qui détruisent la nature par leurs pratiques ancestrales, telles que l'agriculture sur brûlis ou les feux de brousse. L'écobuage ajouté au pastoralisme aurait totalement desséché les sols.

René Maran ne s'inscrit pas dans ce discours décliniste destiné à servir les visées coloniales basées sur la nécessité d'exporter les manières de faire européennes pour augmenter le rendement des terres en Afrique et surtout préserver le capitalisme prédateur.Dans la nouvelle, le rejet de cette organisation du monde et de la façon destructrice d'habiter les lieux est perceptible lorsque le narrateur explique la nécessité de brûler la brousse pour pouvoir cultiver la terre et manger de la viande : "Tuer pour manger, manger pour vivre, il n'était pas de plus belles règles de la vie ${ }^{10}$." Ainsi, après la récolte : «On avait procédé comme de juste, à la récolte du mil rouge et du mil blanc, à celle du maïs et du sésame. La moisson finie et transportée [...] Et maintenant, chaque nuit, de véhéments feux de brousse rongeaient les ténèbres de l'ardeur de leurs incendies. [...] Il voulait chasser ${ }^{11}$.»

Cet épisode sur lequel nous reviendrons dans le cadre de la relation avec l'animal dévoile combien Roger Maran a eu du mal à adopter le point de vue économique et social du colonisateur, qu'il est chargé pourtant de représenter. Sa posture est en avance puisque, de nos jours, il est largement démontré que l'agriculture sur brûlis ne détériore pas plus les sols que l'agriculture intensive.

21 Par là même, Maran l'écrivain rejette l'intensification forcée de l'agriculture, le remplacement des cultures vivrières par les cultures commerciales telle que le caoutchouc.La poḯtique, mère de la fiction, exprime ce que l'administrateur colonial n'a peut-être pas pu dire, contraint au droit de réserve du fonctionnaire.

Mon hypothèse sur un potentiel regard critique du fonctionnaire Maran se fonde sur la note manuscrite qu'il a écrite au laboratoire d'agronomie coloniale au Muséum d'Histoire naturelle après $1924^{12}$, résumé du rapport du "distingué administrateur M. Baudon », et dans laquelle il recopie avec application les passages sur "l'inutilité de la culture du caoutchouc pour l'indigène qui s'épuise à le récolter uniquement pour s'acquitter de l'impôt, système de perception qui constitue une entrave pour le développement du pays (Moyen et Haut-Congo." Il relève mot à mot "le travail harassant pour l'homme qui doit se déplacer à 100 kilomètres de son village ».

De même, des conclusions d'Auguste Chevalier, grande figure des sciences du végétal qui a joué un rôle important dans la politique de la mise en valeur des territoires de l'Empire français, dans la première moitié du $\mathrm{xx}^{\mathrm{e}}$ siècle, Maran retient :

En A.O.F, l'indigène vit mieux, a appris à commercer, ses cultures vivrières se sont étendues. En A.E.F, il en a été malheureusement autrement. Dès 1904, au retour de la mission Chari-Lac-Tchad que nous avions dirigée, nous signalions la gravité de la situation. Nous avons revu en 1912 le Moyen-Congo et le Gabon dans une situation encore plus précaire. Malgré les efforts très méritants de quelques administrateurs, les indigènes étaient obligés de négliger leurs cultures vivrières pour se livrer à la 
récolte du caoutchouc. L'agriculture était en pleine décadence, et la famine sévissait en bien des régions ${ }^{13}$.

Disciple avant l'heure de René Dumont, auteur, en 1962, de L'Afrique noire est mal partie, sa lecture du paysage et de sa mise en valeur souvent destructrice de l'organisation rurale ancestrale est anti-coloniale. Le premier «Goncourt noir » est déjà inquiet et préoccupé par la question environnementale, par son coût humain et écologique.

\section{Poétique}

Postulant que, bien avant l'heure, René Maran a fait sien le concept de «nature ordinaire » né dans les années 1990 et qui définit la nature comme n'excluant pas par principe les actions humaines considérées comme perturbatrices mais les prenant en compte pour le devenir socio-naturel d'un espace - « nature ordinaire » ou nature dont les hommes font l'expérience au quotidien -, je voudrais sans critiquer ou invalider les lectures de Boum et Dog en tant que conte ou fable animalière, étudier la relation de l'auteur à l'animalité dans cette nouvelle qui ouvre le cycle de la Brousse.

Dès le début du texte, René Maran conforte l'approche prométhéenne de la relation entre les humains et les animaux. L'homme doué de raison est le supérieur de l'animal doté d'instinct. L'homme domine et méprise l'animal :

Boum [le nom du chien] signifiait poussière, en parler sara mad-jl'ngaî. De vrai, on ne lui avait pas donné sans raison et il le portait de manière à lui faire honneur. [...] Mais ce n'est pas souvent, hélas! que les chiens et leur géniture trouvent de quoi se réjouir dans la compagnie de l'homme à peau noire. Boum et ses congénères ne savaient que trop qu'on ne récoltait guère, à son service, que buffes ou bourrades, coups de pied agrémentés de coups de trique ou coups de triques corsés de coups de chicotte $^{14}$.

L'humain peut être violent avec l'animal domestique ou sauvage. Il est un prédateur et il chasse.Mais sa violence n'est pas gratuite et l'homme n'est pas naturellement un tueur :

La chasse était moins un jeu qu'une nécessité. Qui voulait manger à sa faim devait chasser. Il ne connaissait pas d'autre moyen de pouvoir à sa nourriture. Telle était la loi de la brousse, la loi de la vie. Chacun pour soi et, pour tous, l'espace, ses terrains plantés d'herbes et d'arbres - et leurs pièges et leurs ressources ${ }^{15}$.

La sentence «Telle était la loi de la brousse, la loi de la vie » met en évidence la filiation scientifique et philosophique que Maran revendique. C'est celle de la lutte pour l'existence, de la struggle for life de Darwin, au cœur de la théorie de l'évolution des espèces, antidote au créationnisme qu'il ne peut admettre en athée déclaré qu'il est.

Ce refus de l'intervention de Dieu dans la création du monde est patent lorsqu'il recourt aux mythes anciens, au panthéon des divinités africaines et de l'animisme dont le rapport à la nature est fort différent pour expliquer la domination naturelle de l'homme bipède sur l'animal quadrupède :

L'homme ne pensait qu'à son semblable, n'avait besoin que de lui, ne s'occupait que de lui. La raison en était des plus simples. L'homme marchait debout, le chien à quatre pattes. L'homme parlait, le chien aboyait. L'homme commandait, le chien craquelait de frissons de peur, obéissait. L'homme était noir, le chien roux ${ }^{16}$.

Néanmoins, tout en confirmant le principe darwinien selon lequel toutes les espèces se reproduisent aussi longtemps qu'elles trouvent des ressources alimentaires et des conditions optimales d'habitat, tout en admettant la loi du plus fort (notamment lors 
du combat entre les deux buffles), Maran s'écarte du père de la théorie de l'évolution en refusant le schéma fondamental de la descendance commune de toutes les espèces et de leur interdépendance.

Donc, l'homme et le chien n'ayant rien de commun, n'étant ni de même race ni de même origine, au chien de se fier à son génie spécifique et de se débrouiller de son mieux, selon ses possibilités propres ${ }^{17}$.

31 Par conséquent, ce passage censé expliquer la lutte pour la vie n'est qu'une légère référence à Darwin.En effet, d'une part René Maran ne reconnait pas la solidarité entre les espèces voulue et clairement exprimée par le scientifique :

Je dois faire remarquer que j'emploie le terme de lutte pour l'existence dans le sens général et métaphorique, ce qui implique les relations mutuelles de dépendance des êtres organisés, et, ce qui est plus important, non seulement la vie de l'individu, mais son aptitude ou sa réussite à laisser des descendants ${ }^{18}$.

D'autre part, Maran fait de la lutte pour la vie un combat entre les espèces et oublie celui qui a lieu au sein des espèces. En ce sens, il s'éloigne sensiblement du naturaliste du XIX siècle.

Mais à sa décharge, une nouvelle n'est pas une rigoureuse démonstration scientifique. L'auteur d'une fiction peut prendre la liberté de se contredire. C'est ce qu'il s'autorise en minant l'irréductible séparation entre la communauté des hommes et celle des animaux. Même si, convenons-en, dans le récit, le groupe animal, le bestiaire, est solidifié par le don du nom de Dog au buffle, qui est le nom du bovin en sara mais signifie chien en anglais, phénomène de gémellité onomastique qui renforce l'idée de complicité entre les quadrupèdes.

Plus précisément, l'écrivain altère la frontière entre l'humanité et l'animalité de deux façons.

En premier lieu, en employant des formes discursives dont l'étude révèle l'écriture canine et bufflesque de Maran. Écriture qui n'est pas réductible à l'utilisation de la figure anthropomorphique du chien Boum et du buffle Dog, et non soutenue par un narrateur hétérodiégétique qui pense, ressent et parle à la place des animaux. En effet, jouant magistralement avec la langue, René Maran crée une poétique de l'attention qui fait de l'animal domestique ou sauvage ce que Donna Haraway nomme une «espèce compagne. »

35 En toute sincérité, Maran choisit « d'habiter la brousse en chien et en buffle ${ }^{19}$ » comme le prouve ce petit inventaire d'exemples non exhaustifs :

36 - Le choix du point de vue, de la perspective : la focalisation est résolument interne, c'est celle des quadrupèdes.

37 - Toute la narration se déroule à hauteur de chien, à raz de terre avec Boum dans la poussière ou dans l'herbe avec Dog.

38 - La description de l'espace, de la brousse s'organise selon deux axes : l'un horizontal et l'autre vertical, axes qui d'ailleurs souvent se croisent.

Horizontalité : description de la faune du bas: les fourmis rouges, les termites, le serpent python, les phacochères. «IL se rasa dans les herbes, s'y carra de son mieux et resta coi.»

Verticalité : « La terre sous eux tremblait. Les oiseaux, les oisillons, les criquets, affolés, s'égaillaient, pèle mêle. Les hautes herbes, sous le poids multiple de sabots quadruples, 
qui éclaboussaient la brousse [...] les hautes herbes croulaient, froissées, arrachées, fracassées, mâchonnées, foulées, piétinées, broyées, déchiquetées ${ }^{20}$. "

Dans ce passage, l'accumulation des participes passés adjectivés permet d'entendre la charge dévastatrice des taureaux.

\section{l'animal :}

Il venait d'entendre, lointains mais distincts, mugissements sur mugissements ${ }^{23}$.

En second lieu, la fable elle-même convoque les " affinités électives entre les espèces » que Darwin ambitionnait de démêler dans son fameux ouvrage, devenu un manuel de compréhension du monde et de son humanité.

Du côté du buffle :

Pas de doute possible. La bête qu'il avait devant lui, et qui le consolait maintenant de son mieux, après lui avoir sauvé la vie, c'était l'une de ces hargneuses bêtes qui parlent le langage ouah-ouah et passent les trois quarts de leur vie aux pieds de l'homme. D'où venait que celle-ci fût seule ? Ce fait le déroutait. Le chien à l'accoutumée ne marche pas sans l'homme. Qui rencontrait l'un ne tardait guère à rencontrer l'autre. Celui-ci tenait lieu de griffes à celui-là, celui-là mettait son flair au service de celui-ci ${ }^{24}$.

Du côté du chien, alors qu'il se pose la question de rester auprès de son ami :

Un pacte immémorial le liait à l'homme. Il n'était rien sans lui. L’Homme le complétait, ou, plutôt il se servait de l'homme, dont il était le serviteur, pour se compléter. Impossible d'hésiter entre Dog et l'homme. Son devoir lui prescrivait de regagner au plus tôt le village où il était né. Force lui était de s'y soumettre ${ }^{25}$.

Les affinités électives ici suggérées insistent sur le rapport asymétrique entre l'homme et l'animal et confortent le rapport de domination.

Mais, tout en affirmant nettement que les affaires « de l'homme ne sont pas affaires de chien ${ }^{26}$ ", Maran aboutit à la réconciliation de l'homme et du chien, tous deux éprouvant la même nécessité et le même désir de chasser :

Boum voulait chasser. [...] randjibaî continuait, comme si de rien n'était, à vaquer à ses travaux habituels. Vraiment, tout cela ne pouvait durer. Et Boum songeait déjà à chasser pour son propre compte, lorsqu'un soir, dans le moment où il se préparait en désespoir de cause à hurler à la lune (instinct), les tambours de Kombra commencèrent à gronder d'une façon qui lui était familière. Boum, aussitôt, se sentit renaître. Les gens de Kombra venaient de se rappeler à temps qu'il fallait manger pour vivre et qu'on ne pouvait manger sans tuer. Tuer pour manger, manger pour vivre, il n'était pas de plus belles règles de vie. La vie dévore les morts, la mort nourrit la vie ${ }^{27}$.

En conséquence, Boum fait son métier de chien, les hommes, leur métier d'hommes en incendiant la brousse et Dog subit courageusement son sort de buffle. À la fin de la nouvelle, chaque protagoniste est réinstallé à sa place donnée au sein de la nature.

Toutefois, en conservant jusqu'au bout de la narration les points de vue du chien auxiliaire de l'homme et du buffle victime logique, Maran refuse tout pathos, n'avance aucune morale et fait dialoguer les espèces. En développant le topos de la chasse, il souligne la parenté sans tomber dans le piège bienpensant de l'anthropomorphisme. Le 
chien et le buffle n'ont pas des comportements humains et l'homme n'est pas bestial. En revanche, est affirmée leur histoire commune, leur coexistence dans le monde des « existants ", ce grand Commun qu'en 2021 il est non seulement nécessaire de protéger mais aussi de réinventer.

\section{Conclusion provisoire}

Par la fiction, Maran met en question, me semble-t-il, la dichotomie entre nature et culture, il nous propose de faire à nouveau connaissance avec l'animal en nous forçant à penser avec lui.

Il me semble que la nouvelle Boum et Dog résiste assez bien à la grille d'analyse de l'écocritique. Reste à poursuivre le travail sur l'ensemble des nouvelles de Bêtes de la Brousse.

\section{BIBLIOGRAPHIE}

Corpus : MARAN, René, Boum et Dog, Les E Euvres libres, n 134, août 1932.

BLANC, Guillaume, L'Invention du colonialisme vert, Paris, Flammarion, 2020.

DARWIN, Charles, L'Origine des espèces au moyen de la sélection naturelle, Paris, C. Reinwald et Cie, Libraires éditeurs, 1873. https://gallica.bnf.fr/ark :/12148/bpt6k77233m.image DESPRET, Vinciane, Habiter en oiseau, Arles, Actes Sud, coll. “Mondes Sauvages, pour une nouvelle alliance", 2019.

HARAWAY, Donna, Manifeste des espèces compagnes, L'Isle-d'Espagnac, Climats, 2019.

LATOUR, Bruno, Face à Gaïa. Huit conférences sur le nouveau régime climatique, Paris, La Découverte, 2015.

\section{NOTES}

1. Définie par Pierre SCHOENTJES dans son ouvrage de référence Ce qui a lieu. Essai d'écopoétique, Wildproject, 2016.

2. Dont son ouvrage Habiter en oiseau, Arles, Actes Sud,2019.

3. René MARAN, Boum et Dog, Les CEuvres libres, n 134, août 1932, p. 5.

4. Gabriel GALLAND, La Conquête du Tchad. Journal de route d'un des membres des deux missions Émile Gentil, Paris, Librairie nationale d'éducation et de récréation, 1907, p. 33.

5. Cet imaginaire anthropophage auquel Maran ne peut adhérer est très présent dans la mentalité des colonisateurs, héros de sa monumentale histoire de L'Empire colonial français intitulée Les Pionniers de l'Empire. Quatre volumes parus chez Albin Michel entre 1943et 1955. Voir Sylvie BRODZIAK, « l'écriture noire de la conquête blanche? » in Interculturel Francophonies, Roger Little ( dir.) numéro consacré à René Maran, Lecce/Italie, 2018.

6. Jean-Pierre TUQUOI, Oubangui-Chari, le pays qui n'existait pas, Paris, La Découverte, 2017, 272 p. 
7. Charles BAUdelaire, «Correspondances », dans Les Fleurs du Mal, première édition 1857.

8. Bruno LATOUR, Face à Gaïa. Huit conférences sur le nouveau régime climatique, Paris, La Découverte, 2015, p. 319.

9. Guillaume BLANC, L'Invention du colonialisme vert. Pour en finir avec le mythe de l'Éden africain, préface de François-Xavier Fauvelle, Paris, Flammarion, 2020, 352 p.

10. René MARAN, Boum et Dog, Les CEuvres libres, n 134, août 1932, p. 27.

11. Ibid., p. 26.

12. Transmise généreusement par monsieur Bernard Michel.

13. P. 27 du rapport de Chevalier, précise René Maran.

14. René MARAN, Boum et Dog, op. cit., p. 6.

15. Ibid., p. 6.

16. Ibid., p. 7.

17. Ibid., p.7

18. Charles DARWIN, L'Origine des espèces au moyen de la sélection naturelle, Paris, C. Reinwald et Cie, Libraires éditeurs, 1873 , p. 67

19. J'imite avec plaisir la belle formule de Vinciane Despret, titre de son ouvrage Habiter en oiseau, op. cit.

20. René MARAN, Boum et Dog, op. cit., p. 13.

21. René MARAN, Boum et Dog, op. cit., p. 9.

22. Ibid., p. 11.

23. Ibid., p. 12.

24. Ibid., p. 17.

25. Ibid., p. 22.

26. René MARAN, Boum et Dog, op. cit., p. 24.

27. Ibid., p. 27-28.

\section{RÉSUMÉS}

L'étude de Boum et Dog pour le projet mené à l'ITEM sur l'œuvre de René Maran s'accomplissant dans une société fortement préoccupée par les questions environnementales invite immédiatement à passer cette nouvelle par le tamis de la critique éco-poétique. Il s'agit donc de voir dans un premier temps si ce texte liminaire résiste à cette grille d'analyse afin de pouvoir l'étendre plus tard au recueil Bêtes de la brousse.

Typing the text of "Boom and Dog" on the computer keyboard for the ITEM René Maran project today, in a society strongly concerned with environmental issues, prompted me immediately to read the tale from an ecopoetic critical perspective. It matters now to see whether this introductory text withstands such an analytical reading, before extending it to all the stories collected in Bêtes de la brousse. 
INDEX

Mots-clés : René Maran, études post-coloniales, écocritique, études animales

Keywords : René Maran, post-colonial studies, ecocriticism, animal studies

\section{AUTEUR}

\section{SYLVIE BRODZIAK}

SYLVIE BRODZIAK est professeure des universités en Littératures française et francophone, Histoire des idées à CY Cergy Paris université, membre de l'UMR-CNRS Héritages. Docteure en Histoire contemporaine, docteure en Langue et Littérature françaises, spécialiste de Clemenceau écrivain, ses recherches en littérature se penchent sur l'expression des groupes et individus dits minoritaires ou marginaux. Elle oriente sa réflexion sur les littératures francophones, l'écriture de l'histoire et des mémoires, les études de genre, l'éco/géopoétique et sur la création littéraire et artistique après les grandes catastrophes historiques, industrielles et naturelles : guerres, génocides, séismes... 